This is the author's final, peer-reviewed manuscript as accepted for publication. The publisher-formatted version may be available through the publisher's web site or your institution's library.

\title{
Luminol-based bioluminescence imaging of mouse mammary tumors
}

Hamad S. Alshetaiwi, Sivasai Balivada, Tej B. Shrestha, Marla Pyle, Matthew T. Basel, Stefan H. Bossmann, Deryl L. Troyer

\section{How to cite this manuscript}

If you make reference to this version of the manuscript, use the following information:

Alshetaiwi, H. S., Balivada, S., Shrestha, T. B., Pyle, M., Basel, M. T., Bossmann, S. H., \& Troyer, D. L. (2013). Luminol-based bioluminescence imaging of mouse mammary tumors. Retrieved from http://krex.ksu.edu

\section{Published Version Information}

Citation: Alshetaiwi, H. S., Balivada, S., Shrestha, T. B., Pyle, M., Basel, M. T., Bossmann, S. H., \& Troyer, D. L. (2013). Luminol-based bioluminescence imaging of mouse mammary tumors. Journal of Photochemistry and Photobiology B: Biology, 127, 223-228.

Copyright: (C) 2013 Elsevier B.V.

Digital Object Identifier (DOI): doi:10.1016/j.jphotobiol.2013.08.017

Publisher's Link:

http://www.sciencedirect.com/science/article/pii/S101113441300198X

This item was retrieved from the K-State Research Exchange (K-REx), the institutional repository of Kansas State University. K-REx is available at http://krex.ksu.edu 


\section{Luminol-based Bioluminescence Imaging of Mouse Mammary \\ 2 Tumors \\ 3}

4 Hamad S. Alshetaiwi ${ }^{a}$, Sivasai Balivada ${ }^{a}$, Tej B. Shrestha ${ }^{a}$, Marla Pyle $^{a}$, Matthew T. Basel $^{a}$, 5 Stefan H. Bossmann ${ }^{\mathbf{b}^{*}}$, Deryl L. Troyer ${ }^{\mathbf{a},{ }^{*}}$

6

$7 \quad{ }^{a}$ Department of Anatomy and Physiology, 228 Coles Hall, Kansas State University, Manhattan, 8 KS 66506, USA.

$9{ }^{\mathrm{b}}$ Department of Chemistry, 213 CBC Building, Kansas State University, Manhattan, KS 66506, 10 USA.

11

12 Hamad S. Alshetaiwi- hamad@ksu.edu

13 Sivasai Balivada- sivasai@ksu.edu

14 Tej B. Shrestha- tbs@ksu.edu

15 Marla Pyle- mpyle@ksu.edu

16 Matthew T.Basel - mbasel@vet.k-state.edu

17 Stefan H. Bossmann- sbossman@ksu.edu

18 Deryl L. Troyer- troyer@vet.ksu.edu

19

20 * Corresponding authors: Deryl Troyer, Department of Anatomy and Physiology, 228 Coles Hall,

21 Kansas State University, Manhattan, KS 66506, USA; email: troyer@vet.ksu.edu, phone: 785-

22 532-4509, fax: 785-532-4557; Stefan H. Bossmann, Chemistry, 201 CBC Building, Kansas

23 State University, Manhattan, KS 66506, USA; email: sbossman@ksu.edu, phone:785-532-6817, 24 fax: 785-532-6666.

25

26

27

28

29

30 


\section{Abstract}

$3 \quad$ Polymorphonuclear neutrophils (PMNs) are the most abundant circulating blood

4 leukocytes. They are part of the innate immune system and provide a first line of defense by

5 migrating toward areas of inflammation in response to chemical signals released from the site.

6 Some solid tumors, such as breast cancer, also cause recruitment and activation of PMNs and

7 release of myeloperoxidase. In this study, we demonstrate that administration of luminol to mice

8 that have been transplanted with 4T1 mammary tumor cells permits the detection of

9 myeloperoxidase activity, and consequently, the location of the tumor. Luminol allowed

10 detection of activated PMNs only two days after cancer cell transplantation, even though tumors

11 were not yet palpable. In conclusion, luminol-bioluminescence imaging (BLI) can provide a

12 pathway towards detection of solid tumors at an early stage in preclinical tumor models.

13

14

15

16

17

18

19

20

21

22

23

24

25

26 


\section{Key words}

3 Bioluminescence, chemiluminescence, neutrophil, luminol, myeloperoxidase (MPO), cancer

4 detection, 4T1 breast adenocarcinoma, murine breast cancer model

\section{Abbreviations}

PMNs- Polymorphonuclear neutrophils, BLI- Bioluminescence imaging, ROS- Reactive oxygen species, MPO- Myeloperoxidase, PBS- Phosphate buffered saline, ROI- Region of interest

\section{Introduction}

Cancer is a major cause of disease and death in the United States and other countries around the world. For example, the American Cancer Society estimates that 580,350 people will die of cancer in the U.S. in 2013 [1]. Detecting tumors at earlier stages could significantly improve therapeutic outcomes [2, 3]. In pursuing this goal, understanding the tumor microenvironment may facilitate tumor diagnosis at the earliest stages. It has been known that tumor microenvironment differs from that of normal tissue in that it is often characterized by low $\mathrm{pH}$, hypoxia, over-expressed proteases [4], and infiltration of defensive and immune cells [5].

These features can potentially be exploited for detection and treatment. Several imaging techniques have been used to examine the tumor microenvironment, such as optical imaging,

22 positron emission tomography (PET) and magnetic resonance imaging (MRI) [4, 6-12]

23 Bioluminescence imaging $(\mathrm{BLI})$ is a non-invasive imaging technique used in pre-clinical oncology research to image tumors by generating visible light, that is usually generated by luciferase-expressing cells $[2,13]$. The luciferase-based BLI mechanism involves the oxidation of luciferin in the presence of adenosine tri-phosphate (ATP), magnesium $\left(\mathrm{Mg}^{2+}\right)$ and molecular oxygen $\left(\mathrm{O}_{2}\right)$ to create an electronically excited oxy-luciferin, which emits visible radiation in the yellow-green to yellow-orange spectrum, with an emission maximum of 560nm [2, 13]. Firefly luciferase transfected mouse mammary gland tumor cells 4T1-luc2 show stable light emission in the presence of luciferin and permit the detection of early tumors [2]. In contrast to luciferasebased BLI mechanism, Liu et al., showed beta galactosidase based chemiluminescent imaging 
for in vivo detection of tumors expressing a beta galactosidase transgene[14] . However,

2 naturally occurring cancers do not express luciferase. Therefore, luciferase-based BLI is not

3 available when treating cancers in humans and/or not transfected tumors in mammals. Note that

4 in the research reported here, luciferase-based BLI is only used to define the region of interest. 5

Luminol (5-amino-2,3-dihydrophthalazine-1,4-dione) is a known chemiluminescent molecule that emits light at maximum of $425 \mathrm{~nm}$ when oxidized [15, 16]. Luminol has been used in various fields, such as biochemistry, analytical chemistry, and clinical diagnostics for detecting reactive intermediates [17]. For instance, luminol has been used as an analytical tool to examine the role of granulocyte-derived reactive oxygen species in heart muscle damage, to screen polymorphonuclear leukocyte function in patients with diabetes mellitus, and to detect leukocyte activity in patients with peritonitis $[15,18]$. Luminol detects phagocytic oxidative bursts and subsequent myeloperoxidase (MPO) activity [17]. Gross et al. have demonstrated that luminol-based imaging can be used for quantitative longitudinal monitoring of MPO activity in animal models of acute dermatitis, mixed allergic contact hypersensitivity, focal arthritis and spontaneous large granular lymphocytic tumors[17]. The work presented here consists of tailoring their detection method for revealing early breast tumors (4T1) in mice. Chronically inflamed tissues like cancer are populated by defensive cells, such as neutrophils, monocytes, and macrophages, which can produce reactive oxygen species (ROS) by respiratory bursts and subsequent chemical reactions. Many of these ROS react with luminol and produce light by means of luminol chemiluminescence [19].Since this chemiluminescence occurs from a chemical and oxidizers that are produced by enzymes in living cells, we will use the term luminol-based bioluminescence imaging (BLI). Here, we present first evidence that administration of luminol to mice that have been transplanted with 4T1 mammary tumor cells permits early stage imaging of tumors by bioluminescence-based detection of MPO activity.

\section{Theory of Luminol-Based Bioluminescence}

When PMNs are activated in inflamed tissues such as tumors, they start releasing myeloperoxidase (MPO) [20, 21]. MPO is an abundant protein produced in azurophilic granules of neutrophils, where it can constitute more than $5 \%$ of the granule's protein [22]. Respiratory burst is initiated by phagocytic NADPH oxidase (Phox). Phox reduces molecular oxygen $\left(\mathrm{O}_{2}\right)$ to 
1 give superoxide anions $\left(\mathrm{O}_{2}{ }^{-}\right)$. In the presence of protons $\left(\mathrm{H}^{+}\right)$, the superoxide anion reacts to the

2 hydroperoxy free radical $\left(\mathrm{HO}_{2}\right)^{\circ}$. Superoxide and hydroperoxy free radicals disproportionate to

3 hydrogen peroxide $\left(\mathrm{H}_{2} \mathrm{O}_{2}\right)$ and molecular oxygen [23]. Superoxide dismutase (SD) accelerates

4 this reaction. During PMN activation, MPO catalyzes the reaction of $\mathrm{H}_{2} \mathrm{O}_{2}$ and chloride ions $\left(\mathrm{Cl}^{-}\right)$

5 to produce hypochlorous acid $(\mathrm{HOCl})$ [17]. In turn, $\mathrm{HOCl}$ can oxidize luminol, which triggers

6 chemiluminescence [17]. MPO can also directly utilize superoxide anion as a substrate for

7 peroxidase-catalyzed oxidation of luminol [17]. The manifold of reactions is shown in scheme 1.

8

Luminol can detect activated neutrophils, which often accumulate in tumor tissue.

Therefore, luminol may be a clinically relevant method for detecting tumors in vivo. To verify the ability of luminol to detect tumors, 4T1 tumors bearing a firefly luciferase gene were generated in Balb/c mice. Luciferin/luciferase is a well-known imaging system that can detect luciferase bearing cells very specifically in vivo. Luciferase detection is clinically irrelevant, though, because clinical tumors do not contain luciferase genes. If luminol imaging could produce images comparable to luciferin in luciferase bearing tumors it would be validated as a tumor detection system. Therefore, luminol bioluminescence was compared to the highly-sensitive but clinically-irrelevant luciferin bioluminescence. We were able to demonstrate using 4T1 tumors in BALB/c mice that MPO detection from neutrophils is especially suited to image tumors very early after implantation.

\section{Material and Methods}

\subsection{Materials and Cell Culture}

Mouse mammary gland adenocarcinoma tumor cell line 4T1luc2 was purchased from Caliper Life Sciences, Hopkinton, MA. This cell line has been engineered to stably express the firefly luciferase gene luc2 [24]. Cells were grown in RPMI1640 medium supplemented with 10\% fetal bovine serum (FBS; Sigma-Aldrich, St. Louis, MO) and 1\% penicillin/ streptomycin (Invitrogen, Grand Island, NY). Cells were incubated at $37^{\circ} \mathrm{C}$ at $95 \%$ humidity in $5 \% \mathrm{CO}_{2}$. Luminol sodium salt was purchased from Gold Biotechnology Inc., St. Louis, MO. Luciferin was purchased from Caliper Life Sciences. 


\subsection{Mouse tumor model}

9 week old female BALB/c mice were obtained from Charles River Laboratories, Wilmington, MA. Mice were maintained according to approved IACUC procedures (3060) in the Comparative Medicine Group facility of Kansas State University. Before 4T1 luc2 cells transplantation, mice fur was clipped on the ventral surface to identify mammary fat pads. $1 \times 10^{5}$ 4T1luc2 cells in $25 \mu \mathrm{l}$ phosphate buffered saline (PBS) were transplanted orthotopically into the fat pad of mammary gland number seven. 4T1luc2 cell line expresses the firefly luciferase gene and tumors can be identified by D-luciferin imaging. Control mice were injected with $25 \mu \mathrm{l}$ phosphate buffered saline (PBS) orthotopically into mammary fat pad number seven.

\subsection{Bioluminescence imaging}

As a positive control, the light sensitive substrate D-luciferin $(15 \mathrm{mg} / \mathrm{mL}$ in $1 X$ PBS) was given to the mice intraperitoneally at a dose of $150 \mathrm{mg} / \mathrm{kg}$. To demonstrate luminol-dependent bioluminescence, a three hour gap was allowed between D-luciferin and luminol injections. After that, mice were injected intraperitoneally with luminol $(30 \mathrm{mg} / \mathrm{mL}$ in water) at a dose of $300 \mathrm{mg} / \mathrm{kg}$. In both cases, mice were placed beneath the CCD camera in an IVIS Lumina II imaging system (Caliper Life Sciences) and retained under isoflurane anesthesia (1.5\%-2.0\%) for the period of imaging. Bioluminescence was recorded 5 minutes after D-luciferin and luminol injections. For D-luciferin, mice were imaged on days 1, 2 and 3 after 4T1luc2 cells or PBS sham injection. For luminol, mice were imaged on days 1, 2, 3, 4, and 5 after 4T1luc2 cells or PBS sham injection. The area of 4T1luc2 cell or PBS injection (mammary pad 7) was selected as the region of interest (ROI) on bioluminescent images (Figure1). Boundaries of the ROI were defined based on the luminescence signal in 4T1luc2 cell-injected mice, and the same size area was used in PBS-injected mice. The average radiance $\left(\mathrm{p} / \mathrm{s} / \mathrm{cm}^{2} / \mathrm{sr}\right)$ from that $\mathrm{ROI}$ was used as measurement for analysis. The duration of detectable bioluminescence occurring from firefly luciferase was found to be 30 minutes after luciferin administration. In order to avoid any potential overlap, we waited three hours after luciferin injection to administer luminol.

\section{Figure1}




\subsection{Luminol injection kinetics}

To determine whether the luminol injection route affects luminescence peak signal time at the tumor site, two mice were injected intravenously with luminol $(30 \mathrm{mg} / \mathrm{mL}$ in water) at a dose of $300 \mathrm{mg} / \mathrm{kg}$. Two other mice were injected intraperitoneally with luminol $(30 \mathrm{mg} / \mathrm{mL}$ in water) at a dose of $300 \mathrm{mg} / \mathrm{kg}$. Both groups were placed beneath the CCD camera of the IVIS system and retained under isoflurane anesthesia (1.5\%-2.0\%) for the period of imaging (55 minutes). Area of $4 \mathrm{~T} 1$ cells or PBS injection (mammary pad 7) on bioluminescent images was selected as the region of interest (ROI). Boundaries of the ROI were defined based on the luminescence signal on 4T1 cells-injected mice, and the same size area was used in PBSinjected mice. The average radiance $\left(\mathrm{p} / \mathrm{s} / \mathrm{cm}^{2} / \mathrm{sr}\right)$ from that ROI was used as measurement for analysis.

\section{Results}

\subsection{D-luciferin imaging}

4T1luc2 cell-transplanted mice showed a bioluminescence signal at mammary fat pad number seven as early as day 1 with D-luciferin imaging (Figure2A), suggesting that with Dluciferin imaging we can detect 4T1luc2 cells before they form palpable tumors. This finding is in agreement with the literature [2]. Note that luciferin bioimaging is used as positive control for luminol imaging. Mice injected with PBS showed no bioluminescence signal with D-luciferin imaging (Figure2A). D-luciferin imaging of mice on days 2 and 3 showed a bioluminescence signal at the 4T1luc2 cell injection site (Figure 2A); the intensity of bioluminescence (average radiance) has an increasing trend over time (Figure2B), indicating that at least during the early days following bioluminescent tumor cell transplantation, an increase in tumor size can be observed by D-luciferin imaging.

Figure 2A,B

\subsection{Luminol imaging}

Bioluminescence imaging after luminol injection in mice showed a bioluminescence signal at the 4T1luc2 cell injection site (mammary fat pad number seven) on all imaged days starting from Day 1 (Figure 3A, red lines). Control mice injected with PBS showed weak signal at the injection site after luminol was administered on Day 1 but no signal on Day 2 (Figure 3A, 
1 blue lines). Apart from the tumor cell injection site, mice showed luminescence at other areas,

2 primarily in the abdominal region. This may be because of peritonitis caused by luminol

3 intraperitoneal injection. Also, some mice showed rectal light (Figure 3A) because luminol is

4 known to be removed from the blood and excreted in the faces and this signal is likely due to

5 this natural excretion. There were also small areas of signal in the neck-head region to skin

6 nicking induced by clipping. Bioluminescence signal intensity from the injected area (mammary

7 fat pad number seven) started increasing 2 days after 4T1luc2 cell transplantation, and over

8 time, the signal intensity increased (Figure 3B). Also, D-luciferin and luminol bioluminescence

9 intensities showed positive correlations on day $1\left(R^{2}=0.84\right)$, day $2\left(R^{2}=0.83\right)$ and day $3\left(R^{2}=\right.$

10 0.82) (Figure 3C).

Figure $3 A, B, C$

\subsection{Luminol injection kinetics}

To see whether there is any difference in bioluminescence peak signal time at the 4T1luc2 cell injection site; mice were imaged after intravenous or intraperitoneal luminol injection. In both cases mice showed peak bioluminescence signal at the tumor area between five and 15 minutes after luminol injection (Figure 4A,B); the signal lasted for one hour after the luminol injection. Consequently, intravenous injection of luminol resulted in the stronger bioluminescence signals. These results are in principal agreement with earlier imaging experiments in mice utilizing firefly luciferase[9, 25]. Since both luminol and luciferin are small molecules, it is not surprising that similar in-vivo kinetics are observed.

Figure 4A, B

\section{Discussion}

The work described above demonstrates that orthotopically transplanted 4T1luc2 mammary adenocarcinoma cells can be successfully imaged by means of luminol-based biolumincescence imaging even before tumors are palpable. Although control mice injected with

31 saline did show a small signal, the control bioluminescence signal disappeared after two days,

32 in contrast to the transplanted tumor signal. Also, mice showed luminescence at other areas

33 apart from the tumor cell injection site, primarily in the abdominal region. This may be because 
of peritonitis caused by intraperitoneal luminol injection. Skin nicks due to clipping also

2 produced small signals in the neck and head region on days 1 and 2 after tumor cell inoculation.

3 The intensity of the luminol-based BLI in mammary fat pad 7 increased over time after tumor

4 transplantation. This is the first report of early tumor imaging of solid carcinomas using luminol

5 alone in a preclinical model without additional components. Previously, luminol-based BLI was

6 shown to be effective in early imaging in a transgenic preclinical model of a hematological

7 cancer [17]. Zhang et al. studied the MPO dependence of the luminol bioluminescence signal

8 [21]. When luminol was injected into MPO-positive mice and MPO-deficient mice, no

9 luminescence signal was observed in the group of MPO-deficient mice. Based on these studies

$10[17,20]$, it is our paradigm that the luminol BLI signal we detected here is based on MPO activity

11 that been released from activated neutrophils because of tumor transplantation [17].

The emission maximum of luminol-based bioluminescence is at $\lambda=425 \mathrm{~nm}[26]$. At this wavelength, tissue penetration (decrease of the luminescence signal to $1 / \mathrm{e}$ ) is estimated at 0.5 $\mathrm{mm}$ [27], corresponding to a tissue path length (99\% light absorption) of approximately $2.2 \mathrm{~mm}$. In conclusion, the penetration depth of luminol-based bioluminescence is not well suited for imaging deep-seated tumors [21]. Therefore, two strategies can, principally, be pursued for achieving enhanced bioluminescence signals. The first strategy comprises measures to enhance the photon flux of the light emitted at $425 \mathrm{~nm}$. It has been reported that $\beta$-cyclodextrin can enhance luminol luminescence [28]. Another study employed the chemiluminescent probe, L-012 (8-Amino-5-chloro-7-phenylpyrido[3,4-d]pyridazine-1,4(2H,3H)dione), a substrate that has the potential of generating more light than luminol, for detecting MPO activity [29, 30]. The second strategy, described by Zhang et al. is using NIR-emitting Q-dot nanoparticles, which absorb the luminol-based bioluminescence at $425 \mathrm{~nm}$ and reemit them as NIR photons [21]. It is noteworthy that the light penetration depth in the near infrared region is approximately 10 times larger than in the blue region of the visible spectrum [27]. The drawback of this strategy is that most nanoparticles of this type are cytotoxic [31].Therefore, administering additional imaging agents, such as NIR-emitting nanoparticles may not be an option when imaging tumors in humans to observe their response to treatment. But, exploring additional measures of enhancing the luminescent signal may be useful for increasing the ability of luminol to detect deeper tumors.

It has been reported that the numbers of neutrophil granulocytes increase in the circulation in animals and humans with a variety of tumors [32].Tumor cells secrete different cytokines and chemotactic factors which can cause recruitment and activation of neutrophils 
and other immune cells. Some examples are interleukins (IL-6 and IL-8) [33, 34], as well as

2 interferons (IFN- $\gamma$, INF- $\alpha$, INF- $\beta$ ), and tumor necrosis factor- $\alpha$ (TNF- $\alpha$ )[35]. Furthermore,

3 neutrophils are known to be a major component of inflammatory infiltrates in glioma [36],

4 micropapillary carcinoma in the pancreas [37], human fibrosarcoma [38] , prostate carcinoma

5 [39] and gastric carcinoma [40]. Also, it has been reported that neutrophils accumulate in the

6 lungs before the arrival of metastatic cells in breast cancer models [41]. These studies, which

7 demonstrate neutrophil infiltration in different types of tumors, suggest that luminol-based BLI

8 detection of infiltrating neutrophils has potential for early human tumor detection.

Luminol has been used in humans for treatment of alopecia areata [42]; those studies showed it to be safe and rapidly excreted, with no toxic side effects observed. In conclusion, luminol or other luminescent substrates could potentially be used in human patients for imaging

12 tumors. Although this study is a proof-of-principle, the method reported here could potentially be 13 developed to screen for recurrence of other superficial cancers.

\section{Acknowledgements}

Hamad S. Alshetaiwi acknowledges financial support from College of Medicine, University of Hail, Saudi Arabia. The authors gratefully acknowledge financial support from National Science Foundation (NSF/CBET, Award \# 0933701 and NSF/DMR, Award \# 1242765) and from the Johnson Cancer Research Center at Kansas State University.

\section{Conflict of Interest}




\section{Figure captions}

Scheme 1: Neutrophil-mediated oxidation and chemiluminescence of luminol (Phox: NADPH oxidase, SD: Superoxide Dismutase, MPO: myeloperoxidase)

Figure 1. Bioluminescence measurement. Mammary pad 7, the site of 4T1luc2 cell injection (red lines, left four mice) or PBS injection (blue lines, right two mice), was selected as the region of interest (ROI). The average radiance $\left(\mathrm{p} / \mathrm{s} / \mathrm{cm}^{2} / \mathrm{sr}\right)$ from that ROI was used as measurement for analysis.

Figure 2. D-luciferin imaging. A D-luciferin injections were given to the mice intraperitoneally on days 1, 2, and 3 after 4T1 luc2 cell injection. Red lines (left four mice): mice injected with 4T1 luc2 cells in mammary fat pad number seven. Blue lines (right two mice): control mice injected with PBS in mammary fat pad number seven. A luminescence signal was observable at mammary fat pad number seven as early as on day 1 when imaging with D-luciferin in 4T1 luc2 cells injected mice. B Graph of the intensity of luminescence (average radiance, $\mathrm{p} / \mathrm{s} / \mathrm{cm}^{2} / \mathrm{sr}$ ) is showing an increasing trend from day 1 to day 3 , suggesting that an increase in tumor size can be observed by D-luciferin imaging, as shown by previous studies[2]. Figure 3. Luminol imaging. A Luminol injections were given intraperitoneally to 4T1-bearing and control groups of mice on days 1 to 5 after 4T1luc2 cell injection or PBS injection. Red lines (left four mice): mice injected with 4T1luc2 cells. Blue lines (right two mice): control mice injected with PBS. Luminol signal was seen at the 4T1luc2 cell injection site (mammary fat pad number seven) on all the imaged days starting from day 1. PBS injected mice (right two mice) showed virtually no signal at mammary fat pad number seven, but showed signal mainly in the abdominal region. B Bioluminescence signal intensity (average radiance $\left(\mathrm{p} / \mathrm{s} / \mathrm{cm}^{2} / \mathrm{sr}\right.$ ) at mammary fat pad number seven increased by 3 days after 4T1luc2 cell transplantation, and over time, the signal intensity increased. C Comparison of D-luciferin and luminol bioluminescence intensities showed positive correlation for day $1\left(R^{2}-0.84\right)$, day $2\left(R^{2}-.83\right)$ and day $3\left(R^{2}-0.82\right)$.

Figure 4. Luminol injection kinetics. A Comparison of intravenous (two mice, right) and intraperitoneal (two mice, left) luminol injections to observe the difference in bioluminescence peak signal time at the 4T1luc2 cell injection site. Mice were imaged every 10 minutes with an accumulation time of 5 minutes, resulting in a total imaging time of $55 \mathrm{~min}$. after luminol

32 injections.. Luminescence peaked between 5 and 15 minutes regardless of injection method. B 
1 Graph showing the bioluminescence signal (average radiance, $\mathrm{p} / \mathrm{s} / \mathrm{cm}^{2} / \mathrm{sr}$ ) intensity after 2 intraperitoneal and intravenous administration.

3

4

5

6

7

8

9

10

11

12

13

14

15

16

17

18

19

20

21

22

23 
[1] R. Siegel, D. Naishadham, A. Jemal, Cancer Statistics, 2012, Cancer Journal for Clinicians, (2013) 11-30.

[2] J.B. Kim, K. Urban, E. Cochran, S. Lee, A. Ang, B. Rice, A. Bata, K. Campbell, R. Coffee, A. Gorodinsky, Z. Lu, H. Zhou, T.K. Kishimoto, P. Lassota, Non-invasive detection of a small number of bioluminescent cancer cells in vivo, PLoS One, 5 (2010) e9364.

[3] S.H. Bossmann, D.L. Troyer, Point-of-care routine rapid screening: the future of cancer diagnosis?, Expert Rev Mol Diagn, 13 (2013) 107-109.

[4] Y. Wu, W. Zhang, J. Li, Y. Zhang, Optical imaging of tumor microenvironment, Am J Nucl Med Mol Imaging, 3 (2013) 1-15.

[5] E.R. Burton, S.K. Libutti, Targeting TNF-alpha for cancer therapy, J Biol, 8 (2009) 85.

[6] M.K. Alhasan, L. Liu, M.A. Lewis, J. Magnusson, R.P. Mason, Comparison of optical and Power Doppler ultrasound imaging for non-invasive evaluation of arsenic trioxide as a vascular disrupting agent in tumors, PLoS One, 7 (2012) e46106.

[7] C.H. Contag, S.D. Spilman, P.R. Contag, M. Oshiro, B. Eames, P. Dennery, D.K. Stevenson, D.A. Benaron, Visualizing gene expression in living mammals using a bioluminescent reporter, Photochem. Photobiol., 66 (1997) 523-531.

[8] I. Lee, O. Hwang, D. Yoo, G. Khang, D. Lee, Detection of hydrogen peroxide in vitro and in vivo using peroxalate chemiluminescent micelles, Bull. Korean Chem. Soc., 32 (2011) 21872192.

[9] Z. Paroo, R.A. Bollinger, D.A. Braasch, E. Richer, D.R. Corey, P.P. Antich, R.P. Mason, Validating bioluminescence imaging as a high-throughput, quantitative modality for assessing tumor burden, Mol Imaging, 3 (2004) 117-124.

[10] S. Sarraf-Yazdi, J. Mi, M.W. Dewhirst, B.M. Clary, Use of bioluminescence imaging to detect enhanced hepatic and systemic tumor growth following partial hepatectomy in mice, Eur J Surg Oncol, 34 (2008) 476-481.

[11] R. Weissleder, S.S. Gambhir, B.D. Ross, A. Rehemtulla, Molecular Imaging: Principles and Practice., in, BC Decker Inc, Hamilton, Ontario, Canada, 2010.

[12] R. Weissleder, M.J. Pittet, Imaging in the era of molecular oncology, Nature, 452 (2008) 580-589.

[13] K. O'Neill, S.K. Lyons, W.M. Gallagher, K.M. Curran, A.T. Byrne, Bioluminescent imaging: a critical tool in pre-clinical oncology research, J Pathol, 220 (2010) 317-327.

[14] L. Liu, R.P. Mason, Imaging beta-galactosidase activity in human tumor xenografts and transgenic mice using a chemiluminescent substrate, PLoS One, 5 e12024.

[15] R. Radi, T.P. Cosgrove, J.S. Beckman, B.A. Freeman, Peroxynitrite-induced luminol chemiluminescence, Biochem J, 290 ( Pt 1) (1993) 51-57.

[16] H. Lundqvist, C. Dahlgren, Isoluminol-enhanced chemiluminescence: a sensitive method to study the release of superoxide anion from human neutrophils, Free Radic Biol Med, 20 (1996) 785-792.

[17] S. Gross, S.T. Gammon, B.L. Moss, D. Rauch, J. Harding, J.W. Heinecke, L. Ratner, D. Piwnica-Worms, Bioluminescence imaging of myeloperoxidase activity in vivo, Nat Med, 15 (2009) 455-461.

[18] L.J. Kricka, X. Ji, 4-Phenylylboronic acid: a new type of enhancer for the horseradish peroxidase catalysed chemiluminescent oxidation of luminol, J Biolumin Chemilumin, 10 (1995) 49-54.

[19] W.T. Chen, C.H. Tung, R. Weissleder, Imaging reactive oxygen species in arthritis, Mol Imaging, 3 (2004) 159-162.

[20] T. Franck, S. Kohnen, G. de la Rebiere, G. Deby-Dupont, C. Deby, A. Niesten, D. Serteyn, Activation of equine neutrophils by phorbol myristate acetate or $\mathrm{N}$-formyl-methionyl-leucyl- 
phenylalanine induces a different response in reactive oxygen species production and release of active myeloperoxidase, Vet Immunol Immunopathol, 130 (2009) 243-250.

[21] F.K. Zhang N, Prakash A, Ansaldi D., Enhanced detection of myeloperoxidase activity in deep tissues through luminescent excitation of near-infrared nanoparticles., Nature Medicine, 19 (2013) 500-505.

[22] S.J. Klebanoff, Myeloperoxidase: friend and foe, J Leukoc Biol, 77 (2005) 598-625.

[23] S.I. Liochev, I. Fridovich, Superoxide and iron: partners in crime, IUBMB Life, 48 (1999) 157-161.

[24] E. Lim, K.D. Modi, J. Kim, In vivo Bioluminescent Imaging of Mammary Tumors Using IVIS Spectrum, J Vis Exp, (2009) e1210.

[25] Y. Inoue, S. Kiryu, K. Izawa, M. Watanabe, A. Tojo, K. Ohtomo, Comparison of subcutaneous and intraperitoneal injection of d-luciferin for in vivo bioluminescence imaging, Eur. J. Nucl. Med. Mol. Imaging, 36 (2009) 771-779.

[26] L.J. Kricka, Clinical applications of chemiluminescence, Analytica Chimica Acta, 500 (2003) 279-286.

[27] T.B. Shrestha, G.M. Seo, M.T. Basel, M. Kalita, H. Wang, D. Villanueva, M. Pyle, S. Balivada, R.S. Rachakatla, H. Shinogle, P.S. Thapa, D. Moore, D.L. Troyer, S.H. Bossmann, Stem cell-based photodynamic therapy, Photochem Photobiol Sci, 11 (2012) 1251-1258.

[28] R. Maeztu, G. Tardajos, G. Gonzalez-Gaitano, Natural cyclodextrins as efficient boosters of the chemiluminescence of luminol and isoluminol: exploration of potential applications, J Phys Chem B, 114 (2010) 2798-2806.

[29] S. Ahmed, N. Kishikawa, K. Ohyama, T. Maki, H. Kurosaki, K. Nakashima, N. Kuroda, An ultrasensitive and highly selective determination method for quinones by high-performance liquid chromatography with photochemically initiated luminol chemiluminescence, J Chromatogr A, 1216 (2009) 3977-3984.

[30] S. Yamaguchi, N. Kishikawa, K. Ohyama, Y. Ohba, M. Kohno, T. Masuda, A. Takadate, K. Nakashima, N. Kuroda, Evaluation of chemiluminescence reagents for selective detection of reactive oxygen species, Anal Chim Acta, 665 (2010) 74-78.

[31] M. Mahmoudi, K. Azadmanesh, M.A. Shokrgozar, W.S. Journeay, S. Laurent, Effect of nanoparticles on the cell life cycle, Chem Rev, 111 (2011) 3407-3432.

[32] S.A. DuPre, K.W. Hunter, Jr., Murine mammary carcinoma 4T1 induces a leukemoid reaction with splenomegaly: association with tumor-derived growth factors, Exp Mol Pathol, 82 (2007) 12-24.

[33] A. Mantovani, P. Allavena, A. Sica, F. Balkwill, Cancer-related inflammation, Nature, 454 (2008) 436-444.

[34] R. Bonecchi, M. Locati, A. Mantovani, Chemokines and cancer: a fatal attraction, Cancer Cell, 19 (2011) 434-435.

[35] E. Di Carlo, G. Forni, P. Lollini, M.P. Colombo, A. Modesti, P. Musiani, The intriguing role of polymorphonuclear neutrophils in antitumor reactions, Blood, 97 (2001) 339-345.

[36] N.A. Atai, M. Bansal, C. Lo, J. Bosman, W. Tigchelaar, K.S. Bosch, A. Jonker, P.C. De Witt Hamer, D. Troost, C.A. McCulloch, V. Everts, C.J. Van Noorden, J. Sodek, Osteopontin is upregulated and associated with neutrophil and macrophage infiltration in glioblastoma, Immunology, 132 (2011) 39-48.

[37] S. Khayyata, O. Basturk, N.V. Adsay, Invasive micropapillary carcinomas of the ampullopancreatobiliary region and their association with tumor-infiltrating neutrophils, Mod Pathol, 18 (2005) 1504-1511.

[38] M. Shimizu, T. Yoshimoto, M. Sato, J. Morimoto, A. Matsuzawa, Y. Takeda, Roles of CXC chemokines and macrophages in the recruitment of inflammatory cells and tumor rejection induced by Fas/Apo-1 (CD95) ligand-expressing tumor, Int J Cancer, 114 (2005) 926-935. [39] E.M. Bekes, B. Schweighofer, T.A. Kupriyanova, E. Zajac, V.C. Ardi, J.P. Quigley, E.I. Deryugina, Tumor-recruited neutrophils and neutrophil TIMP-free MMP-9 regulate coordinately 
1 the levels of tumor angiogenesis and efficiency of malignant cell intravasation, Am J Pathol, 179 2 (2011) 1455-1470.

3 [40] R.A. Caruso, R. Bellocco, M. Pagano, G. Bertoli, L. Rigoli, C. Inferrera, Prognostic value of 4 intratumoral neutrophils in advanced gastric carcinoma in a high-risk area in northern Italy, Mod 5 Pathol, 15 (2002) 831-837.

$6 \quad$ [41] Z. Granot, E. Henke, E.A. Comen, T.A. King, L. Norton, R. Benezra, Tumor entrained

7 neutrophils inhibit seeding in the premetastatic lung, Cancer Cell, 20 (2011) 300-314.

8 [42] S. Irie, The treatment of alopecia areata with 3-aminophthal-hydrazide, Curr Ther Res Clin 9 Exp, 2 (1960) 107-110. 